\title{
Second-Language Reading Process for Professional English: A Case Study
}

\author{
Che-Han Chen ${ }^{1}$ \\ ${ }^{1}$ Department of Teaching and Learning, Ohio State University, Columbus, Ohio, United States of America \\ Correspondence: Che-Han Chen, Department of Teaching and Learning, Ohio State University, Arps Hall, 1945 \\ N. High St., Columbus, Ohio 43210, United States of America.
}

Received: August 8, 2021

Accepted: August 27, 2021

Online Published: August 30, 2021

doi: $10.5539 /$ elt.v14n9p80

URL: https://doi.org/10.5539/elt.v14n9p80

\begin{abstract}
Although much research on English for specific purposes (ESP) has been conducted, little focus has been placed on investigating the mechanisms involved in ESP readers' comprehension process. This case study explored the reading process that characterizes a competent ESP reader's comprehension. A juris doctor student attending a major university in the United States was selected for the case study. Qualitative data were gathered through a semi-structured interview, recall protocol, and document analysis, and were thematically coded. The findings revealed the following: (a) The professional domain determines the major purposes of ESP reading. (b) Institutional demands and inadequate L2 proficiency within the target discourse prevent background knowledge in the first language (L1) from being transferred to L2 texts are the major sources of comprehension difficulty. (c) Three strategies can be used to overcome such difficulties. First, schemata (background knowledge) in L1 can facilitate comprehension and enhance the reading process. Second, genre knowledge learned in L1 can be used to dissect the information structure of L2 texts. Finally, the use of L1-based materials can help to overcome the comprehension difficulties arising from the L2 material. The implications for L2 reading instruction and future ESP reading research are discussed.
\end{abstract}

Keywords: reading process, reading strategies, reading difficulties, English for specific purposes, metacognition, qualitative research method

\section{Introduction}

Research on English for specific purposes (ESP) has attracted scholars in applied linguistics and Teaching English to Speakers of Other Languages (TESOL) for years. The primary concerns for ESP are to produce linguistically competent language users to approach academic or occupational goals within a specialized field. Literature has claimed that reading occupies a central role in English for general purposes (EGP), and reading is often considered the skill with which students are the most familiar (Jordan, 1997). However, research indicated that the process underlying EGP reading comprehension are often considered a separate research focus from the reading research in ESP, and is therefore in need of further investigations (Amirian, 2013).

ESP reading has been studied since the 1970s, when the primary focus was on register analysis. The goal was to explore the pure linguistic structures used in a particular domain, such as science or aviation industry (Hutchinson \& Waters, 1987). In particular, the attention was paid to study the lexicon and sentence-level of the use of English language in various communication settings. Subsequently, the research shifted to the study of the discoursal elements within a specialized text, which could lead the way ESP readers process and understand the target texts (Trimble, 1985; Widdowson, 1979). This changing tide of research, according to Hirvela (2013), had the major pedagogical implication that the primary goal of ESP reading instruction is to equip students with the ability to identify and make use of the discoursal devices within the texts and extract relevant information with the needs to read in varying contexts. Current ESP reading research centers on investigating whether ESP reading instruction can link the learning of reading skills to the rhetorical and communicative situations in which the target texts are embedded (Belcher, 2006; Paltridge, 2012). Based on this conceptual framework, ESP reading literature has seen increasing numbers of scholars exploring the effects of genre-based approach and discourse analysis on ESP readers' acquisition of professional reading competence (Hirvela, 2013; Northcott, 2013; Sadeghi et al., 2013). 
While most ESP reading research has identified the contextual and linguistic natures of these highly specialized texts and the pedagogical implications arising from these investigations, little evidence is produced to shed lights on elucidating the elements and cognitive mechanisms underlying ESP readers' reading process (Amirian, 2013). As pointed out by Jordan (1997), whenever a reader starts to read, there is a purpose that governs readers' decision making and choices of reading strategies. Such phenomenon was also observed by Koda (2004, p. 204):

"Accomplished readers continuously adjust their reading behaviors to accommodate text difficulty, task demands, and other contextual variables. Monitoring their reading process carefully, they take immediate steps when encountering comprehension problems. Aware of their own cognitive and linguistic resources, they are capable of directing their attention to the appropriate clues in anticipating, organizing, and retaining text information."

Because of the complexities and components that form accomplished readers' reading behaviors, the purpose of the present study was to investigate what characterizes a competent ESP reader and the process through which ESP readers set goals of reading, identify the sources of reading difficulties, and decide which reading strategies to employ to overcome comprehension problems when they read complex and professional texts.

\subsection{Research Questions}

In order to explore competent ESP readers' reading behaviors and comprehension process, this study investigated the following research questions by qualitatively gathering the data regarding a bilingual ESP reader's reading process:

1) What are the reading purposes generally perceived by ESP readers when they read the highly specialized materials?

2) What are the sources of difficulties that hinder an ESP reader's reading process?

3) How does such conceptualization of reading difficulties affect an ESP reader's choice of reading strategies?

\subsection{Literature Review}

This section provides literature review about three strands in Second-Language (L2) reading research: reading process, reading difficulties, and reading strategies. Because previous literature about reading comprehension process for specialized English was based largely on reading for general English (Hirvela, 2013), this review included research in L2 reading and selected aspects that are relevant to the ESP context.

\subsubsection{Reading Process}

Reading process has been built around understanding the comprehension process that involves successful use of core reading skills and the ability to analyze discourse features of a given text. Anderson (2008) indicated that successful reading process entails the process by which the readers demonstrate mindful, fluent, and active engagement with the texts, through which the readers can construct the meaning from target materials. Reading research has proposed a variety of model attempting to elucidate the patterns underlying reading process, and most of them are characterized as bottom-up, top-down, and interactive ways for readers to process information. According to Koda (2004), these reading models explain how readers' cognitive mechanisms work through using diverse reading strategies while processing complex materials. Acknowledging the complexity involved in reading process, Grabe (2009) specified a set of component skills that are necessary for a reader to build effective comprehension of a text:

1) recognizing words efficiently, such as phonological, orthographic, morphological awareness

2) a wide range of grammar and vocabulary knowledge

3) discourse and genre knowledge that allows readers to know how information is structured

4) appropriate use of background knowledge (schema)

5) proper execution of working memory abilities (short-term memory)

6) ability to formulate main idea, synthesize information, and make inference

7) using metacognitive strategies that allow readers to monitor their cognitive resources when processing challenging texts according to the goals of reading (reading to learn, reading for general comprehension, expeditious reading, etc.), text types (genres), and reading tasks assigned to them

In addition to psycholinguistic approach to understanding the cognitive processes through which competent readers instantiate these core reading components in order to tackle complex texts, as seen in the sociocultural 
turn in second language acquisition (SLA) research, reading process has started to include contextual and social factors that are also thought to mediate the reading process (Bernhardt, 2005). For example, Lee and Chern's qualitative study (2011) investigated whether there is a relationship between a EFL learner's identity formation and reading process in ESP. Analyzing through Wenger's (1998) community of practice and learners' social identity, they concluded that ESP readers' reading development is tightly interwoven with their identity construction. Consistent with Bernhardt's (2005) assertion, Lee and Chern's research has provided an alternative perspective that comprehension process depends on not only the execution of various cognitive operations, but also the negotiation between the reader's sociocultural experiences and written words.

The aforementioned constructs of reading have been proven to be general characteristics of what an accomplished reader display, and these factors also provide a comprehensive picture of what a reader's reading behavior look like at various linguistic proficiency levels and under different reading purposes (Grabe \& Jiang, 2014). Nevertheless, as indicated earlier, this well-established framework was mainly conducted in reading for general purposes. As a result, questions remain as to how and to what extent can these concepts be applied to understand readers with specialized needs, and how such needs to read texts within an academic or professional discourse community affect ways that readers utilize these reading abilities in ESP context.

\subsubsection{Reading Difficulties}

Another strand of reading research investigates reading difficulties readers often encounter during the comprehension process. Horwitz et al. (1986) suggested that past literature concerning the most significant factor impeding foreign language (FL) acquisition is the concept of foreign language anxiety, particularly with respect to oral performance in classroom and assessment settings. Drawing on this perspective, Saito et al. (1999) conducted a preliminary study of 30 first-semester foreign language courses of Spanish, Russian, and Japanese in a university. Their finding suggested that the source of reading difficulties arise from the following two factors: (1) unfamiliar writing systems, which would interfere ways that reader decode the sound-symbol of the texts and subsequent construction of meaning; (2) cultural knowledge embedded in the target texts, in which the lack of cultural knowledge or contextual awareness of the target language would prevent readers from constituting comprehensible message after he or she has successfully decoded the printed symbols. Based on this finding, they introduced the construct of foreign language reading anxiety and proposed a rating scale to measure the psychometric properties underlying such cognitive difficulty in $\mathrm{L} 2$ reading process.

In addition to feeling anxious while reading in a L2, Hauptman (2000) reviewed empirical and pedagogical literature regarding another source of L2 reading difficulty and found that traditional view of difficulty in L2 reading lies in linguistic deficiency and text length. In a similar vein, Koda (2004) indicated linguistic threshold as major factor for successful L2 reading achievement, suggesting that the inadequate L2 proficiency can not only interfere readers to effectively decode sound-symbol in the texts, but also prevent readers from activating background knowledge that, based on Hauptman's (2000) term called schema, compensate for linguistic deficiency during the comprehension process.

\subsubsection{Reading Strategies}

Once the reading difficulties have been identified by the readers, accomplished readers may use different reading strategies that are appropriate for the types of texts they are reading. Grabe (2009) indicated the use of metacognition as an essential element of reading strategy, asserting that successful readers should be able to regulate and control their cognitive process. Such awareness and regulation of their cognitive resources can allow readers to plan, monitor, repair, and evaluate their comprehension based on their goals of reading and reading tasks. This will allow readers to use such understanding to decide the kind of strategies to enhance comprehension of difficult texts.

Within metacognitive approach to enhancing reading comprehension, research also shows that being familiar with text types and genre knowledge can allow readers to read more strategically (Grabe, 2009; Hirvela, 2013). Richards and Schmidt (2010) defined genre as "a type of discourse that occurs in a particular setting, that has distinctive and recognizable patterns and norms of organization and structure, and that has particular and distinctive communicative functions." (p. 224) With regard to reading strategy development, Sadeghi et al. (2013) explored the effects of genre-based reading instruction could have on ESP students' reading comprehension of Iranian college students majoring in biology. Their findings suggested that genre awareness had a significant role in facilitating ESP learners' reading comprehension. Consistent with this finding, Camiciottoli (2003) recommended that teachers should help students identify the discoursal patterns used in disciplinary texts because this can allow ESP readers to selectively extract relevant information and discard what is insignificant based on their reading purpose, and therefore construct an appropriate mental representation of the texts. 


\section{Methodology}

\subsection{The Participant}

The case study focused on Cathy, a Taiwanese graduate student. She is a proficient bilingual reader, with Chinese as her first language (L1) and English as her second language (L2), and is pursuing a juris doctor (JD) degree at a university in the United States. Two criteria were used to determine that Cathy was a competent L2 English reader: (a) the result of a qualitative four-item self-report questionnaire adapted from Martinez's (2008) college reading research and (b) the highest score she achieved on the TOEFL iBT reading section prior to her arrival in the United States. The self-report questionnaire required Cathy to rate her L2 reading proficiency on a scale of 1 (poor) to 4 (mastery) in response to the following statements: "When I read in English, I can understand the main point of the texts"; "When I read in English, I can understand and summarize the texts I have read"; "When I read in English, I can understand the structures and most details of the texts"; and "When I read in English, I can understand, synthesize and make inference about the ideas of the texts." Cathy reported her reading ability in legal contexts as mastery. In addition, to complement her self-assessment which was subject to potential bias, I considered her highest TOEFL reading score, 29, which, according to ETS' latest performance descriptors for the TOEFL iBT test (2019), is indicative of an advanced level of academic English reading proficiency. The aforementioned pre-interview assessments helped to conclude Cathy to be a competent L2 English reader.

Cathy acquired two master of laws, one in her native country, Taiwan and the other one in the United States, prior to joining the JD program at the same university in the United States. During the interview, she reported that in law school, the readings assigned by the professors are extensive, typically several hundred pages each week. For each week's class session, students are expected to read at five or more cases and are asked to answer related questions (either through "on call," by which teacher systematically pick students or through "cold calling", by which teacher randomly select students). In addition, she has extensive writing-oriented assignments, which increase her weekly workloads as she also needs to handle each week's intensive reading schedules. Cathy had extensive experiences in reading and writing about law-related materials in her L1 and L2, and presented a master's thesis in her L1 in Taiwan.

\subsection{Materials}

The materials for this study were the textbooks that the participant found the most difficult to read and understand: Case Study of Criminal Law, the U.S. Constitution, and Introduction to Patent Law. Case Study of Criminal Law, which describes the occurrence of criminal events and is followed by the court's opinion within a specific law context, was her most used material. The court's opinion that emerges from such cases is inducted into a specific legal item in an assigned case study. In addition to the court opinion that is the ultimate decision made by the court, which is subsequently inducted into the specific law item, some cases provide either the dissenting opinion (disagreement with the ultimate sentence and the application of the legal item) or concurring opinion (agreement with the ultimate sentence and the application of the law but disagreement with the court interpretation and application). Furthermore, legal texts are characterized by highly inaccessible language, including diction and sentence structures, which, according to Cathy, is written to be understood only by those with legal expertise.

\subsection{Data Collection}

In reading research, several methodologies are commonly employed. In this study, I employed two qualitative research methods. The first one was the recall protocol. Using this method, the researcher asks a series of questions based on the research objectives and record the responses with the oral consent. During the interview, I asked Cathy to elaborate on how she read the legal texts she provided, including the purpose she perceived of reading, real-life constraints that made her reading process arduous, and strategies she employed to overcome such reading difficulties. The recall protocol method enables a researcher to obtain first-hand information regarding how participants read designated texts, assuring that the data are sufficiently reliable and authentic for further analysis. The second research method I employed was document analysis. In this method, the researcher collects and examines the artifacts of the participant's reading materials such as their handwritten or electronic notes or other designated texts, to further analyze participants' written interactions with the source texts and the way they organize and process the information while reading the complex materials.

\subsection{Data Analysis Process}

The data gathered through the recall protocol and the document analysis regarding the participant's overall reading process were recorded and transcribed. I thematically coded the transcripts with patterns typical of an 
ESP reader's reading difficulties and the general conceptualization of reading strategies in response to identified comprehension obstacles. These classifications were based on literature regarding metacognitive approach and strategies of L2 reading (Grabe, 2009; Koda, 2004), reading difficulties (Saito et al., 1999; Hauptman, 2000; Koda, 2004), schema-based reading (Hauptman, 2000), and genre-based reading (Sadeghi et al., 2013). This is because their theoretical frameworks and empirical evidence provide the general guidelines to categorize the response I obtained during the interview while coding the data. In the discussion section, I organize my interpretations primarily in the form of answering the research questions posted earlier, with a secondary focus on identifying the emerging themes that were originally not anticipated or accounted for by the research questions but I believed might broadly characterize strategic reading in the ESP context.

\section{Findings and Discussions}

In this section, I describe the patterns of Cathy's reading process for law English. Specifically, I interpret the findings with reference to the concepts of strategic reading identified by Grabe (2009) and Koda (2004); reading difficulties mentioned by Saito et al. (1999), Hauptman (2000), and Koda (2004); schema-based reading proposed by Hauptman (2000); and genre-based approach supported by Sadeghi et al. (2013). Accordingly, I attempt to answer the research questions posed earlier and reveal the features that generally characterize competent ESP reading.

\subsection{Research Question 1: What are the Reading Purposes that are Generally Perceived by ESP Readers When They Read the Highly Specialized Materials}

As a JD student, Cathy faced highly demanding reading workloads and expectations set by her law professors. She stated that she had been under such demands since her master's study in the United States and that they only increased in the JD program. In the initial stage of the interview, Cathy reflected on the types of discussions and the modes of lectures in a regular law class:

In each class meeting, all the professors would start their class session by asking s series of questions regarding the case study we read for the week. Some professors prefer to use the "on call method," which is to pick students based on the order of the students list, or "cold calling method," which means the professors would randomly pick a student to answer whatever questions he or she asks. The most terrifying mode of discussion is "cold calling" because to be sure you can provide the responses that satisfy the professors, you literally need to have a firm grasp of the reading materials. And unfortunately, most professors in our law school prefer "cold calling" way more than "on call" to ask students questions.

In addition to the needs to answer the questions posted through these methods by law professors, Cathy also mentioned that the examinations and writing assignments throughout her JD study also served as the major purpose to engage herself with arduous reading tasks as imposed by her status as a law professional and the need to meet law community's expectations in the in-class discussions.

Asides from the purpose of fulfilling her coursework requirements, Cathy also read to pass the bar examination and to become a qualified lawyer. She perceived that the heavy reading workload in the law school was generally considered as a part of the school's role as "a lawyer training center"; that is, all pre-service lawyers are expected to model themselves toward what it means to be a lawyer, judge, or legal consultant. She commented on the purpose of reading the law materials:

To me, the nature of law is all about a process of interpretation and application of a certain law item. The major purpose for me to read the law materials right now is to provide me with a chance to understand the existing law concepts and items and to use the cases that happened in the past to predict for the future and imagined contexts where the law item could be applied. I believe I can draw upon these concepts once I finish my study here and step into the workplace.

During the initial part of the interview, the recurring theme in Cathty's reading story is the strong connection between her identiy as a future legal expert within the law community and how she conceptualized the purpose of reading. As pointed out by Grabe's (2009) article, metacognitive ability is an essential element of being a strategic reader, and that the first element involved in such ability is to set and evaluate a goal of reading before the action of reading. In Cathy's case, she managed to identify contextual factors (as a JD student and a future legal expert) that largely shaped her understanding of the importance of reading specialized law materials. To be specific, Cathy's ability to be aware that competent legal English readers should use speed reading skills such as skimming and scanning and identify discourse markers typical of legal discourse helps her locate specific information more efficiently. As a result of connecting the contextual awareness to how she should process the 
information in the legal texts, Cathy used this understanding to help her gain a clearer sense of how contexts of reading shape her reading purpose and subsequently decide which reading strategies to employ in order to fulfill the goals of reading and make the reading process move forward.

\subsection{Research Question 2: What are the Sources of Difficulties that Hinder an ESP Reader's Reading Process}

The previous section revealed that competent ESP readers should possess the ability to identify factors that shape their goals of reading before they actually read. However, the texts read by most ESP readers are generally characterized by their highly specialized and inaccessible language as well as other contextual factors that pose challenge and interfere with an ESP reader's reading process (Northcott, 2009, 2013). As Cathy indicated, law school is often typified by its high demands in terms of reading assignments, in-class discussions, and meeting professors' expectations. She reported that throughout her LLM and JD study, reading was never an easy task; she has constantly struggled to read efficiently and effectively:

The workload of reading in the law school is extremely heavy to me. In each week's reading assignment, professors do not seem to realize how many pressures and burdens students would have by asking us to read at least several hundred pages. But the truth is I have many subjects to take right now, so in the beginning of my study I was always overwhelmed by these crazy amounts of reading homework.

The nature of ESP reading requires learners to efficiently extract the necessary information in the specialized texts and to use such information to complete work in a particular domain (Hirvela, 2013). However, as exemplified by Cathy's case, ESP readers are usually unable to read every word on the paper because of time constraints. Coupled with the "cold calling method" employed by her professors as an explicitly perceived reading stress, the construct of foreign language reading anxiety is also observed in Cathy's reading experience. That is, the need to read in order to answer professors' questions in front of classmates and read under time constrained elicited uncomfortable and intimidating feelings that prevented Cathy from mindfully processing linguistic structures in English and activating the prior knowledge stored in her L1, thereby increased Cathy's perceptions of text difficulty. This is consistent with Saito et al.'s (1999) finding that the highly inaccessible language (writing system) and the contextual factors (emotional reactions as a result of external environment) that are thought to hinder an ESP reader's comprehension process.

Another factor concerning the reading difficulty that ESP readers usually encounter is the lack of proper background knowledge. In reflecting the difference between reading Criminal Law and the U.S. Constitution, Cathy recounts:

In the textbooks we use for the current study, I prefer to read Criminal Law (name of a specific textbook) than the Constitution due to its fundamental differences of the law system and how the cases are structured. In addition to this, some compilers of the textbooks would also affect my reading preference because of their way and preference to organize the textbook. Some are easy to comprehend while others are not.

Grabe (2009) indicated that certain genres or the nature of texts could affect the way a reader reads them. Cathy's preference to read Criminal Law over the U.S. Constitution arose from the fact that she had studied law in Taiwan. While reading the materials, she discovered many similarities between Taiwanese criminal law and the U.S. counterpart; therefore, she could reference what she had learned about criminal law in Taiwan (schema) and transfer that knowledge to the L2 context. However, the constitutions of Taiwan and the United States differ conceptually; thus, no clear connection between the contexts could help her bridge the gap to comprehend the information embedded in the target legal discourse, in this case, the U.S. Constitution. Such schema-based reading in relation to the readers' perception of reading difficulties has also been empirically demonstrated in Hauptman's article (2000) that activating domain knowledge of the target texts can help readers compensate linguistic deficiency and facilitate comprehension process.

Notably, because the focal ESP reader is a bilingual language user, the relationship between two language systems is also involved in the reading process. Cathy elaborated on why she often felt a sense of low confidence while processing law texts in English:

Sometimes I feel like the American classmates in my class do not work really hard in terms of reading the required materials. Yet, they are always able to answer the questions during the "on call" and "cold calling" sessions and also manage to finish the writing assignments more easily than I. As for me, probably because English is my L2, I have to spend more time on reading the materials in order to extract the information. American folks can go to parties on the weekends, whereas I have to stay at home to study in order to catch and compete with them. 
In the context of L2 reading, insufficient L2 proficiency could stand as a major obstacle that prevents a bilingual reader like Cathy from reading like a native speaker. Cathy's L2 proficiency affected the way she reads the texts by slowing her reading rate, making reading the materials under the given time constraints unlikely; therefore, she was unable to both complete the assigned reading and establish the proper level of coherence to fulfill the purposes of reading. This linguistic threshold, according to Koda (2004) and Hauptman (2000) can impede readers to build appropriate mental representation of the texts because inadequate L2 proficiency can not only interfere readers to effectively decode sound-symbol in the texts, but also prevent readers from activating prior knowledge that compensate for linguistic deficiency during the comprehension process and subsequent construction of meaning.

\subsection{Research Question 3: How does Such Conceptualization of Reading Difficulties Affect ESP Readers' Choice of Reading Strategies}

In addition to discussing how competent ESP readers identify their purpose of reading in relation to the contexts and the general sources of reading difficulty when processing information in highly specialized materials, it is imperative to explore what reading strategies does an accomplished ESP reader typically employ to overcome the perceived obstacles while reading difficult texts.

\subsubsection{Reading Strategy 1: Using Proper Background Knowledge (Schema-Based Reading)}

According to Hauptman (2000) and Nassaji (2007), the first indicator of a text's difficulty to L2 readers is whether they have the proper schema and background knowledge from which to draw during the reading process. As Cathy mentioned earlier regarding the preferred genres to read, when she began reading a new case study in Criminal Law, she could resort to her background knowledge and the schemata of the criminal law she had studied back in Taiwan (L1 schemata) and draw upon the similarities between the two legal systems to help her comprehend the target texts more easily. This theme of a schema-based reading approach to new case studies continually emerged during the recall protocol and document analysis of Cathy's reading behaviors. As a result, the appropriate use of background knowledge might indicate a pattern of reading strategy that a competent ESP reader generally employ while tackling the specialized texts.

\subsubsection{Reading Strategy 2: Grasping How the Information is Structured When Schema-Based Reading Fails}

However, findings of Cathy's narrative showed that there were occasions where transfer of L1 schema to L2 reading was not observed when she read the U.S. Constitution, and in such case, schema-based reading did not always effectively work in all reading processes. This arose from the lack of clear connections between her understanding of the Taiwanese constitution when she read the US counterpart. This lack of domain knowledge, based on Saito et al.'s (1999) concept called cultural knowledge of the target texts, hindered her reading process and rendered such texts difficult for her to read fluently (Hauptman, 2000). Thus, if schema-based approach did not work and when time constraints were imposed, she came up with a strategy to overcome the challenge:

Whenever the time is short, I would read the court opinion first because that's the majority's opinion which will always be inducted to a specific law item in that chapter and is very frequently asked by professors during the "on call" and "cold calling" sessions. Thus, it should be read more thoroughly. After reading that part, I will decide if I have to read either dissent or the concurrence opinion based on the time remaining because that is usually considered as supplementary information.

By determining which sections to be read first and the attention sources to be paid in terms of how the information is structured in the law discourse, Cathy managed to construct the proper standard of coherence to meet the expectations and purposes of reading in a particular domain (Grabe, 2009). She also pointed out the importance of locating the abstract section at the beginning of a case; in a sense, the information therein would enable her to build a general understanding of how the texts should be read and processed. In particular, such overviews usually have a general framework of how the case and court opinion is structured, which in turn allowed her to effectively construct the overall meaning of the texts and ultimately achieve the goal of reading. This finding is corroborated by the work of Sadeghi et al. (2013), who provided empirical evidence that genre-based approach has a significant role in enhancing an ESP reader's comprehension process.

In addition, when background knowledge was unavailable, Cathy used online resource called Case Brief, a study guide produced by other students from her law school to help process information of law texts. By using the key points from Case Brief that highlighted information structure of legal texts, she could navigate the long and dense legal passages more efficiently as well as to select the relevant information that is aligned with the expectations and purposes of her reading. As the case study demonstrates, to maximize the effectiveness of tools such as web-based resources, an ESP reader must have a firm grasp of how the information is arranged in the 
target discourse at first, and how to read strategically by directing their own cognitive and linguistic resources to the appropriate clues in anticipating, organizing, and retaining text information.

\subsubsection{Reading Strategy 3: Power of L1 in L2 ESP Reading}

Given the focal ESP reader was a bilingual reader, it is conceivable that there would be the interaction between two language systems while she was engaged in L2 reading (Koda, 2004). During the interview, Cathy revealed that L1 serves as a powerful tool that helps her process numerous law-related terms that are difficult to understand, even without clear correspondence between English term and the Chinese. Using this word processing strategy, she managed to understand messages in the L2 texts by drawing from both linguistic and non-linguistic resources (schema of the Taiwanese legal system) to facilitate reading specialized L2 texts (Hauptman, 2000; Koda, 2004).

L1 also helped Cathy to take effective notes while processing L2 information. For instance, when reading a L2 case study, because of her insufficient L2 proficiency, Cathy found retaining every detail under time constraints extremely difficult, such as the names of the participants involved, where the events of the case occurred, the relevant legal items to be applied, and the title of the case. However, paradoxically, her L1 attrition was transformed into a tool that she could use to overcome the comprehension difficulties arising from L2 issue. To be specific, using her L1 to craft customized name tags for each case after reading it as linguistic cues, Cathy managed to translate the legal English texts into her native language and used L1-based working memory to summarize and organize the reading materials. Such use of her L1 helped her maintain a proper standard of coherence by being cognizant of the rhetorical features that are commonly used in English legal discourse. This in turn informed her to decide which parts of the texts to be read with more attention and which sections to be scanned and left out trivial details, which facilitated overall comprehension process by being aware of the cognitive resources being allocated while reading (Grabe, 2009; Koda, 2004). As a result of this use of strategy, Cathy was able to not only selectively locate relevant information and the major points in a specific case study or other law-related texts, but also managed to retain, synthesize, and make inferences when the reading process was concluded, which in turn enabled her to use the information embedded in conceptually dense L2 texts in accordance with her reading purposes.

\section{Conclusion}

The findings of the present study demonstrate the general reading patterns of a competent ESP reader's reading behavior. Such process begins by setting a specific goal before reading, identifies the possible sources of reading difficulties, and ultimately decides which reading strategies to employ to facilitate comprehension and to make the reading process move forward. In determining the purposes of a reading activity, an ESP reader needs to pay attention to the contextual factors in which the purposes established by the target community. Cathy's perceived reading purpose is largely depended on her awareness as a JD student and her imagined identity as a future lawyer within law profession. During the reading process, Cathy's constantly encountered numerous reading difficulties such as a lack of necessary background knowledge, time constraints, and the insufficient L2 proficiency that slowed her reading. However, she managed to devise various strategies to overcome such difficulties and achieve her goals of reading. The implication of this study is that, to counteract perceived reading difficulties, ESP readers should enrich and familiarize themselves with the background knowledge in their professional domains (schema-building) and the linguistic features that characterize the target genres (genre-based reading). Moreover, ESP readers should increase their L2 proficiency to enable them to smoothly navigate the specialized texts and locate relevant information effectively (linguistic threshold). In addition, it is crucial that the strategic reading is of huge importance in ESP reading and that should be more rigorously addressed in today's English instruction to provide students with robust skills for coping with this unique reading activity.

\section{References}

Amirian, S. M. R. (2013). Teaching reading strategies to ESP readers. International Journal of Research Studies in Educational Technology, 2(2), 19-26. https://doi.org/10.5861/ijrset.2013.318

Belcher, D. D. (2006). English for specific purposes: Teaching to perceived needs and imagined futures in worlds of work, study, and everyday life. TESOL Quarterly, 40, 133-156. https://doi.org/10.2307/40264514

Bernhardt, E. (2005). Progress and procrastinating in second language reading. Annual Review of Applied Linguistics, 25, 133-150. https://doi.org/10.1017/S0267190505000073

Camiciottoli, B. C. (2003). Metadiscourse and ESP reading comprehension: An exploratory study. Reading in a Foreign Language, 15(1), 28-44. https://doi.org/10125/66586 
Grabe, W. (2009). Reading in a second language: Moving from theory to practice. Cambridge: Cambridge University Press. https://doi.org/10.1017/CBO9781139150484

Grabe, W., \& Jiang, X. (2014). Assessing reading. The Companion to Language Assessment, 185-200. https://doi.org/10.1002/9781118411360.wbcla060

Hauptman, P. C. (2000). Some hypothesis on the nature of difficulty and ease in second language reading: An application of schema theory. Foreign Language Annual, 33, 622-631. https://doi.org/10.1111/j.1944-9720.2000.tb00931.x

Hirvela, A. R. (2013). ESP and reading. In Paltridge, B. \& Starfield, S., (Eds.), The Handbook of English for Specific Purposes (pp. 77-94). Hoboken, NJ: Wiley. https://doi.org/10.1002/9781118339855.ch4

Horwitz, E. K., Horwitz, M. B., \& Cope, J. A. (1986). Foreign language classroom anxiety. Modern Language Journal, 70, 125-132. https://doi.org/10.1111/j.1540-4781.1986.tb05256.x

Hutchinson, T., \& Waters, A. (1987). English for Specific Purposes: A learning-centred approach. Cambridge, UK: Cambridge University Press. https://doi.org/10.1017/CBO9780511733031

Jordan, R. R. (1997). English for academic purposes: A guide and resources book for teachers. Cambridge: Cambridge University Press. https://doi.org/10.1017/CBO9780511733062

Koda, K. (2004). Insights into second language reading: A cross-linguistic approach. Cambridge: Cambridge University Press. https://doi.org/10.1017/CBO9781139524841

Lee, B. C., \& Chern, C. (2011). ESP reading literacy and reader identity: A narrative inquiry into a learner in Taiwan. Journal of Language, Identity, and Education, 10(5), 346-360. https://doi.org/10.1080/15348458.2011.614547

Martinez, A. C. L. (2008). Self-reported reading strategy use among Spanish university students of English. Spanish Journal of Applied Linguistics, 21, 167-179.

Nassaji, H. (2007). Schema theory and knowledge-based processes in second language reading comprehension: A need for alternative perspectives. Language Learning, 72(1), 79-113. https://doi.org/10.1111/0023-8333.00189

Northcott, J. (2009). Teaching legal English: Contexts and cases. Ann Arbor, MI: University of Michigan Press.

Northcott, J. (2013). Legal English. In Paltridge, B. \& Starfield, S. (Eds.), The Handbook of English for Specific Purposes (pp. 213-226). Hoboken, NJ: Wiley. https://doi.org/10.1002/9781118339855.ch11

Paltridge, B. (2013). Genre and English for specific purposes. In Paltridge, B. \& Starfield, S. (Eds.), The Handbook of English for Specific Purposes (pp. 347-366). Hoboken, NJ: Wiley. https://doi.org/10.1002/9781118339855.ch18

Richards, J. C., \& Schmidt, R. (2010). Longman Dictionary of Language Teaching and Applied Linguistics. London: Routledge.

Saito, Y., Horwitz, E. K., \& Garza, T. (1999). Foreign language reading anxiety. The Modern Language Journal, 83(2), 202-218. https://doi.org/10.1111/0026-7902.00016

Sedeghi, B., Hassani, M. T., \& Hemmati, M. R. (2013). The effects of genre-based instruction on ESP learners' reading comprehension. Theory and Practice in Language Studies, 3(6), 1009-1020. https://doi.org/10.4304/tpls.3.6.1009-1020

Trimble, L. (1985). English for science and technology: A discourse approach. Cambridge: Cambridge University Press.

Widdowson, H. G. (1979). Explorations in applied linguistics. Oxford: Oxford University Press.

\section{Copyrights}

Copyright for this article is retained by the author(s), with first publication rights granted to the journal.

This is an open-access article distributed under the terms and conditions of the Creative Commons Attribution license (http://creativecommons.org/licenses/by/4.0/). 